\title{
ESTIMATION OF EXPOSURE ZONE BOUNDARY DISTANCES FROM FAR-FIELD ANTENNA CHARACTERISTICS FOR FINITE RADIUS WIRE ANTENNA ARRAYS
}

\author{
Harald Ebersbach*', David V. Thiel' ${ }^{1}$ and Mark Leckenby ${ }^{2}$ \\ 'Radio Science Laboratory, Griffith University, Nathan, QLD 4111, Australia \\ ${ }^{2}$ iCOMMS Pty Ltd, Mitchelton, QLD 4053, Australia
}

I. Introduction; Increasingly, wireless communication network operators are required to demonstrate compliance with RF exposure limits imposed by local authorities via measurement or numerical calculation of the occupational and non-occupational exposure zone boundary distances from the antenna sites. Computational techniques are sought for accurate and efficien calculation of the near-fields of all antenna structures on a wireless communication tower.

The problem for existing computational techniques is the often limited available physical specifications of the antennas at the site. In the transmitter databases of the Australian Communications Authority, Telstra and iCOMMS [1], the only information stored is the antenna class (such as wire, aperture or panel antenna), gain and front to back ratio, far-field radiation patterns in both principal planes, and its maximum physical dimensions. This is insufficient for present NEC, FDTD and FEM software tools, which require complete mechanical specifications of the antenna structure. Thus an additional pre-calculation step is required, that infers an antenna structure from the available antenna specifications.

This paper presents an algorithm for estimating the occupational and non-occupational exposure zone boundaries of ill-defined, multi-element wire antennas. From the gain and half-power beamwidths in both principal far-field radiation planes, an estimate of the number of elements in the antenna array is obtained. This estimate is used to select a reference antenna with consequently similar radiation characteristics to the original antenna to be modelled. Its nearfield radiation characteristics are then modelled using an improved and extended version of the Huygen's wavelets technique for Yagi-Uda and multi-element wire antennas [2]. Results of the implemented $\mathrm{C}++$ algorithm compared to NEC [3] show good accuracy for tested dipole and Yagi-Uda antenna arrays.

II. Antenna Structure Estimation: Green [4] has compiled a large antenna database of rumerically derived design data for short to medium length Yagi-Uda antenna arays, including different antenna designs with varying number of antenna elements, element spacings and conductor diameters. The forward gain, front-to-back ratio, input impedance, half-power beamwidth and first null and side-Jobe intensity and position in both principal radiation planes was also included in the database, providing a source of theoretical data for rapid design of Yagi-Uda antenna arrays. For the purpose of this paper, Green's antennas were analysed to investigate the relationship between each antenna's radiation characteristics and physical design properties.

Fig. 1 shows the relationship between the forward gain and the number of antenna elements of the Yagi-Uda antenna array. As can be seen, the antenna gain increases monotonically with an increase in the number of antenna elements. Also displayed in Fig. 1 are the half-power beamwidths in the $\mathrm{E}$ - and $\mathrm{H}$-planes of the antenna far-field radiation pattern for varying number of antenna elements, these beamwidths decreasing with increasing antenna element number. Additionally it was also observed that since Green's antenna designs were optimized for maximum gain, the relationships of gain and half-power beamwidths with the number of antenn elements shown in Fig. 1 were independent of the conductor diameter used, ard varied only slightly with different element spacings.

0-7803-8302-8/04/\$20.00 C2004 IEEE 
While the half-power beamwidths are easily obtainable from the antenna E- and H-plane farfield radiation patterns and the gain is also available from the central antenna database, these parameters can be employed to acquire a good estimate of the number of antenna elements in the Yagi-Uda antenna array to be modelled for a reasonably well designed antenna.

From the estimated number of antenna elements a suitable reference Yagi-Uda antenna design is selected from a database. The same number of elements should give sirnilar radiation pattem characteristics. For the purpose of this paper, the antenna design data by Green [4] could again be used to design a number of antennas to be utilized within the catalogue of reference antennas. The chosen reference antenna design may then be used to represent the original antenna to be modelled, and its near- and far-field radjation characteristics and exposure zone boundaries computed.

III. Near-Field Modelling: To determine the occupational and non-occupational exposure zone boundaries of the antenna, it is necessary to model the radiation contour from the near-field extending into the far-field.

While antenna engineers and manufacturers are typically most interested in the far-field radiation characteristics, methods to efficiently calculate the far-field of wire antennas are well represented in the literature and are included in most fundamental electromagnetic and antenna textbooks $[5,6]$. Calculation of the near-fields however requires a more involved and laborious analysis. Modelling algorithms that have been shown to accurately calculate the near-field of wire antennas, including the method of moments and finite difference time domain techniques $[3,6]$, are generally difficult to set up and are computationally expensive.

One approximate method that has been shown to efficiently model the near-field of infinitely thin wire antennas with reasonable accuracy is the Huygen's wavelets technique for Yagi-Uda and wire antennas, with radiating point sources placed at current maxima and minima [2]. This method has now been extended to wire antennas with finite radius elements, and improved to give higher agreement with equivalent NEC and measured Yagi-Uda antennas.

Based on the mutual emf-method [6], Baker and LaGrone [7] have preserted a numerical technique for computing the complex mutual-impedance between thin dipoles of any length and geometrical configuration that is easily solvable by today's desktop computers. This algorithm is used, to calculate the mutual-impedances between all antenna array elements.

The effect of modelling antenna arrays with finite radius elements, may be compensated while calculating the self-impedances of the wire antenna elements. According to Baker and LaGrone, the self-impedance of a thin wire antenna element may be simulated as the mutual-impedance between two identical elements with very close spacing [7]. Due to the inherent nature of the underlying mutual enf-method of the Baker and LaGrone algorithm, it has been found that by setting this spacing to the element radius, the self-impedances of such finite radius dipoles may be obtained with close conformance to impedance data obtained by other numerical techniques and measured results $[6,8]$.

From the mutual- and self-impedances, irritial current magnitudes are calculated using the network equations [6]. The input impedance $Z_{I N}$ is then the ratio of the applied voltage to the current in the driven element. For a specified transmitter input power $P_{I N}$, the input voltage $V_{I N}$ of the driven element can be calculated. Using the true input voltage $V_{I N}$ then yields the complex current amplitudes of each respective wire antenna element by re-solving the network equations.

The current distribution of each wire is then replaced by one or more radiating point source placed at the locations of the current maxima and minima. The complex E-field in the near- or far-field is computed by superposition of the complex E-ficld contributions of each point source. 
IV. Results: The described algorithm was implemented in $\mathrm{C}++$ and applied to several dipole and Yagi-Uda antenna arrays. Amongst the antennas tested was a known six-element Yagi-Uda antenna [5], whose far-field radiation in both principal planes has been modelled using NEC and is shown in Fig. 2(a). This antenna has a NEC forward gain of $11.28 \mathrm{dBi}$, while its half-power beamwidths are $38^{\circ}$ and $43^{\circ}$ in the E- and $\mathrm{H}$-planes respectively.

From the lookup curves of gain and half-power beamwidths (Fig. 1), the number of elements of the original antenna is estimated to be 6 elements from the gain curve and 7 elements for each of the E- and H-plane beamwidth curves. This indicates that the original 6-element antenna is best approximated by the 7-element antenna of the reference antenna database. The NEC modelled far-field radiation pattern of the 7-element reference antenna has been plotted in Fig. 2(b).

Utilizing the Huygen's wavelets algorithm for Yagi-Uda and wire antennas, the near-field of the 7-element antenna was then modelled. Initially, calculating the mutual- and self-impedances using the Baker and LaGrone algorithm and assuming sinusoidal current distributions for each antenna element, the input impedance of the antenna was calculated as $Z_{I N}=24.96+j 1.612 \Omega$. From the maximum transmitting power of the antenna, $P_{I N}=50 \mathrm{~W}$, the excitation voltage $V_{I N}$ was calculated, being equal to $35.34+\mathrm{j1.140} \mathrm{V}$. Finally, the complex current amplitudes of each antenna array element were calculated.

While all antenna elements of the reference antenna were shorter than $0.5 \lambda$ each wire could be represented by just a single radiating point source. Utilizing Huygen's wavelets principal, the near-field of the antenna was modelled within an area of $10 \lambda \times 10 \lambda$. The obtained E-field radiation contour is shown in Fig. 3(a).

According to ARPANSA [9] the reference levels for time averaged exposure to RMS electric fields for occupational and non-occupational categories for $410 \mathrm{MHz}$ are $62.16 \mathrm{~V} / \mathrm{m}$ and 27.74 $\mathrm{V} / \mathrm{m}$ respectively. These were highlighted in the computed near-field field-strength contour of the 7-element reference antenna and are shown alongside NEC based calculations for the 6element original antenna in Fig. 3(b)

V. Conclusion: A method of estimating the occupational and non-occupational exposure zone boundaries of ill-defined multi-element wire antennas, utilizing only the antenna forward gain and half-power beamwiđths in both principal far-field radiation planes has been presented. Upon choosing a suitable reference antenna with similar radiation characteristics to the original antenna to be examined, the near-field and exposure zones could be efficiently calculated using Huygen's wavelets technique for multi-element wire antennas. While not providing an exact solution, this algorithm does offer an efficient solution with high accuracy compared to NEC especially when only limited information of the antenna to be modelled is available.

Acknowledgments: The authors would like to thank iCOMMS Pty Ltd and the Australian Research Council for their financial support of this project.

References

[1] iCOMMS Pty Ltd have further information on transmitter databases.

[2] H. Ebersbach and D.V. Thiel, "Yagi antenna modelling using a number of radiating point sources," $8^{\text {th }}$ Australian Symposium on Antennas, CSIRO Sydney, p. 36, February 2003.

[3] Lawrence Livermore National Laboratory, "Numerical Electromagnetics Code (NEC)," Livenmore, CA

[4] H.E. Green, "Design Data for Short and Medium Length Yagi-Uda Arrays," Elec. Eng. Trans. Inst. Engs. Australia, pp. 1-8, March 1966.

[5] D.K. Cheng, Field and Wave Electromagnetics $-2^{\text {nd }}$ Edition, Addison-Wesley Publishing Company, Reading, MA, 1989.

[6] C.A. Balanis, Antenna Theory and Design $-2^{\text {nd }}$ Edition, John Wiley \& Sons Inc., New York, NY, 1997. 
[7] H.C. Baker and A.H. LaGrone, "Digital Computation of the Mutual Impedance Between Thin Dipoles," IRE Trans. Antennas Propagat., vol. 10, pp. 172-178, March 1962.

[8] R.W.P. King, The Theory of Linear Antennas with Charts and Tables for Practical Applications, Oxford University Press, London UK, 1956.

[9] Australian Radiation Protection and Nuclear Safety Agency (ARPANSA), Radiation Protection Standard: Maximum Exposure Levels to Radiofrequency Fields $-3 \mathrm{KHz}$ to 300 $G H z$, ARPANSA, Yallambie, Australia, May 2002.

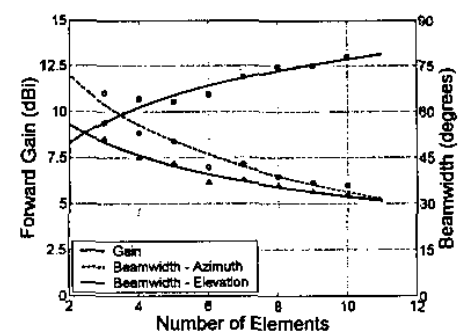

Fig. 1. Lookup curves for gain and half-power beamwidths

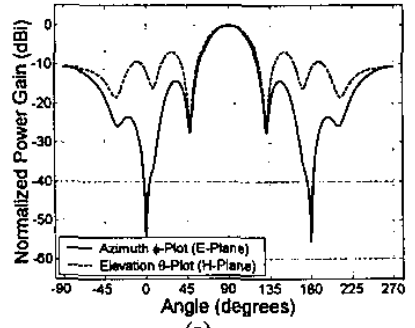

(a)

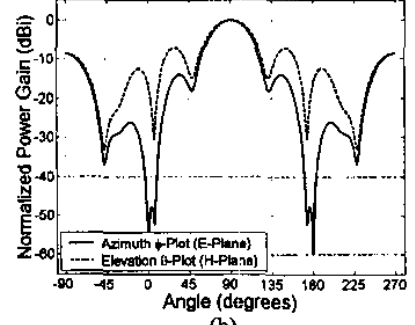

(b)

Fig. 2. Far-field radiation pattern of the original antenna (a) and reference antenna (b)

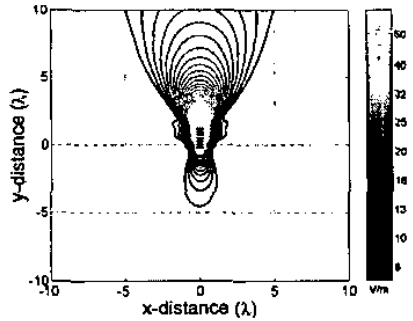

(a)

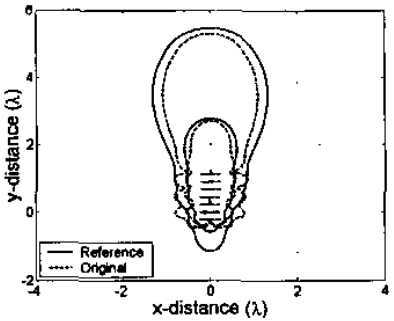

(b)

Fig. 3. (a) Near-field field-strength contour of the 7-element Yagi-Uda reference antenna calculated using Huygen's wavelets technique; and (b) Occupational (inner lines) and nonoccupational (outer lines) E-field exposure zone boundaries calculated using Huygen's wavelets technique (solid lines) and NEC (dashed lines) 